\title{
Cartographie et éducation populaire
}

Le Musée Cartographique d'Élisée Reclus et Charles Perron à Genève (1907-1922)

Cartografia e educação popular. O Museu Cartográfico de Élisée Reclus e Charles Perron em Genebra (1907-1922)

Cartografía y educación popular. El Museo Cartográfico de Élisée Reclus y

Charles Perron en Ginebra (1907-1922)

Cartography and popular education. The Cartographic Museum by Élisée Reclus and Charles Perron in Geneva (1907-1922)

\section{Federico Ferretti}

\section{OpenEdition}

\section{Journals}

Édition électronique

URL : https://journals.openedition.org/terrabrasilis/178

DOI : $10.4000 /$ terrabrasilis. 178

ISSN : 2316-7793

Éditeur

Rede Brasileira de História da Geografia e Geografia Histórica

Référence électronique

Federico Ferretti, «Cartographie et éducation populaire », Terra Brasilis [En ligne], 1 | 2012, mis en ligne le 06 novembre 2012, consulté le 05 décembre 2022. URL : http://journals.openedition.org/ terrabrasilis/178; DOI : https://doi.org/10.4000/terrabrasilis.178

Ce document a été généré automatiquement le 5 décembre 2022

Tous droits réservés 


\section{Cartographie et éducation populaire}

Le Musée Cartographique d'Élisée Reclus et Charles Perron à Genève (1907-1922)

Cartografia e educação popular. O Museu Cartográfico de Élisée Reclus e Charles Perron em Genebra (1907-1922)

Cartografía y educación popular. El Museo Cartográfico de Élisée Reclus y

Charles Perron en Ginebra (1907-1922)

Cartography and popular education. The Cartographic Museum by Élisée Reclus and Charles Perron in Geneva (1907-1922)

\section{Federico Ferretti}

\section{NOTE DE L'ÉDITEUR}

Ofeceremos neste mesmo número uma versão portuguesa deste artigo sob o título Cartografia e educação popular. O Museu Cartográfico de Élisée Reclus e Charles Perron em Genebra (1907-1922).

Ofrecemos en este mismo número una versión portuguesa de este artículo con el título Cartografia e educação popular. O Museu Cartográfico de Élisée Reclus e Charles Perron em Genebra (1907-1922).

Nous offrerons dans cet même número une version portuguaise de cet article sous le titre Cartografia e educação popular: O Museu Cartográfico de Élisée Reclus e Charles Perron em Genebra (1907-1922).

We offer in this same number a Portuguese translation of this paper under the title Cartografia e educação popular. O Museu Cartográfico de Élisée Reclus e Charles Perron em Genebra (1907-1922). 


\section{Introduction}

Le recueil d'environ 10.000 cartes laissé à la Bibliothèque de Genève par le géographe anarchiste Élisée Reclus (1830-1905) et par son cartographe et compagnon de militance Charles Perron (1837-1909) constitue une source exceptionnelle, et encore peu exploitée, pour l'histoire de la cartographie. On y trouve les originaux des cartes dessinées par Perron, entre 1875 et 1894, pour la Nouvelle Géographie Universelle (dorénavant NGU) ainsi que les brouillons, épreuves et notes de travail qui permettent d'analyser la «fabrique " du monumental ouvrage reclusien en 19 volumes. Mais on y trouve surtout des cartes de tout genre, du $16^{\mathrm{e}}$ au $20^{\mathrm{e}}$ siècle (y compris les reproductions des plus célèbres cartes de l'antiquité et du moyen âge), recueillies pendant des décennies par les deux géographes comme source pour leur ouvrage encyclopédique, et dont une sélection a constitué le socle du Musée Cartographique de la Ville de Genève. ${ }^{1}$

2 Cette institution, ouverte en 1907 sous la direction de Perron après le mort de Reclus et après plus de quinze ans de négociations, se lie explicitement à l'idée d'éducation populaire, laïque, scientifique et progressiste pour laquelle les anarchistes de l'époque côtoient parfois les républicains et les libéraux les plus avancés. Cette démarche s'applique à la fois aux jeunes en âge scolaire et aux adultes des classes populaires. Le musée, malgré un certain succès public, fermera ses portes en 1922, mais ses matériaux cartographiques, ainsi que les études dont ils avaient fait l'objet, ont survécu.

Dans l'historiographie contemporaine, les musées ouverts à cette époque sont considérés comme des monuments et des hauts-lieux des États nationaux : quels sont les enjeux d'un musée projeté par des auteurs clairement hétérodoxes et financé par une institution un peu spéciale, c'est-à-dire la ville républicaine de Genève ? Un musée cartographique, que signifie-t-il pour des géographes qui sont à la fois les fondateurs du mouvement anarchiste international? La critique de la carte bidimensionnelle, constante dans leur œuvre, quel rôle y joue-t-elle?

4 Pour répondre à ces questions nous analyserons, à l'aide de la littérature existante, le riche fonds cartographique déposé à la Bibliothèque de Genève et les articles et brochures où Reclus et Perron abordent les problèmes de l'éducation populaire, de l'enseignement de la géographie et de l'emploi de cartes, globes et reliefs dans cette tâche. Nous nous focaliserons particulièrement sur les documents concernant le musée, son catalogue, et l'implication d'institutions locales comme la municipalité genevoise et la Société de Géographie de Genève (dorénavant SGG).

\section{La genèse du musée : géographie et éducation populaire}

5 Le Musée Cartographique de la Ville de Genève, ouvert entre 1907 et 1922 dans le bâtiment des Bastions, qui abrite encore aujourd'hui la Bibliothèque de Genève et son Département des Cartes et Plans, est le résultat de l'engagement persévérant et volontariste du cartographe et militant anarchiste genevois Charles-Eugène Perron, qui s'est battu pour son institution auprès de la SGG et des institutions genevoises. Cet engagement commence en 1891, alors qu'Élisée Reclus, après son retour en France, 
laisse sa collection de cartes en Suisse, où il travaillait depuis 1872 à une grande entreprise : la rédaction de la monumentale NGU.

Perron a été le cartographe de ce travail collectif ${ }^{2}$ qui a vu la publication de plus de 6.000 cartes, qui se rapprochent de ce qu'aujourd'hui on appelle la «cartographie thématique ». Pour la production de ces images, Reclus et Perron s'appuyaient sur des sources de tout genre, envoyées par leurs correspondants de chaque coin du globe, dans leur souci de disposer des données les plus à jour possible sur chaque lieu étudié dans leur ouvrage. Perron présente ce recueil cartographique à une séance de la SGG, en précisant qu'il contient les

cartes, plans et reliefs topographiques qui ont servi à la préparation des seize volumes déjà parus de la NGU. Cette collection unique comprend plus de 6000 cartes. Il y en a peu qui soient de curiosités bibliographiques ou historiques; mais ce sont généralement les meilleures cartes modernes que l'on possède, ce qui a une très grande valeur pour les géographes pratiques. Puis le mérite de cette collection est encore rehaussé par la présence d'un grand nombre d'esquisses ou levés originaux envoyés directement à $\mathrm{M}$. Reclus. $\mathrm{M}$. Charles Perron déclare qu'il met cette riche collection à la disposition de ses collègues, membres de la SGG, qui pourront la visiter et la consulter chez lui, en attendant qu'il ait pris les mesures nécessaires pour la rendre accessible à un public plus nombreux. ${ }^{3}$

7 Le propos de faire connaître ce patrimoine cartographique à un public plus vaste que celui des spécialistes est fortement enraciné dans la démarche géographique reclusienne. Élisée Reclus et ses collaborateurs (Perron, mais aussi d'autres géographes anarchistes comme Léon Metchnikoff et Pierre Kropotkine) ne sont pas des géographes académiques : ils vivent principalement de leur plume, et leur capacité d'atteindre le grand public est à la fois une nécessité matérielle et un choix politique déclaré. En effet, ces scientifiques s'encadrant dans la démarche évolutionniste de leur époque sont aussi des militants, donc l'idée que la géographie puisse être utile à l'éducation populaire (des enfants et des adultes à la fois) est centrale dans leur démarche.

Dans les milieux des militants de la Fédération Jurassienne, section suisse de l'Internationale antiautoritaire qui se sépare des marxistes en 1872 , on trouve les premières affirmations du mouvement de la pédagogie libertaire, qui sera très répandu en Europe dans les décennies suivantes. ${ }^{4}$ En 1868, Perron publie à Genève le pamphlet De l'obligation en matière d'instruction, où il affirme clairement son idée de la centralité stratégique de la diffusion de l'instruction publique pour la tâche du progrès social et de la transformation de la société. «L'ignorance, voilà le vice social organique, la cause première du désordre! C'est là qu'il faut frapper, et frapper fort; car si l'on fait disparaître cette lèpre, la vraie, la dernière révolution sera accomplie $»^{5}$. En 1876 Reclus et Perron constituent à Vevey une section de la Fédération Jurassienne, qui édite le journal Le Travailleur, où ils souhaitent la création d'écoles libertaires et d'universités populaires, où la géographie trouvera bientôt une place prépondérante. Comme l'affirment les membres de cette section,

nous sommes bien loin de nous être assurés l'instruction qui nous est nécessaire pour lutter avec avantage contre les oppresseurs. Par une sanglante ironie du sort, c'est à eux qu'il nous faut demander ce que nous apprenons. La plupart d'entre nous sont encore forcés d'envoyer leurs enfants dans des écoles où des hommes, aux gages de la bourgeoisie, travaillent à pervertir le bon sens et la morale en enseignant non les choses de la science, mais les fables impures du christianisme, non les vertus de l'homme libre, mais les pratiques de l'esclave. ${ }^{6}$ 
Malgré le lieu commun qui les envisage comme des "utopistes", les anarchistes de cette époque développent pour ce but des démarches assez pragmatiques, jusqu'à côtoyer parfois les républicains et les libéraux engagés dans la construction de l'éducation primaire laïque. Donner aux fils des classes populaires la possibilité de s'alphabétiser en se passant de l'éducation confessionnelle est alors une priorité pour les intellectuels libertaires : c'est pour cela qu'on explique, en France, la collaboration directe d'anarchistes comme James Guillaume et Paul Robin avec Ferdinand Buisson pour son Dictionnaire de Pédagogie et pour d'autres expériences pédagogiques financées directement par le Ministère de l'éducation publique. ${ }^{7}$

Charles Perron, lors de ses démarches auprès de la SGG et des pouvoirs publics genevois, ne fait autre chose que s'adresser à la bourgeoisie libérale locale, en nom d'un projet pédagogique populaire et "scientifique", ce qui à l'époque signifie d'abord «non religieux». L'un des membres les plus célèbres de la SGG, correspondant de Reclus et titulaire de la première chaire de géographie à Genève, William Rosier, sera l'un des principaux soutiens du projet de Perron. Ce géographe est aussi l'un des dirigeants du parti radical genevois, et comme le soulignent ses biographes, son engagement pour l'éducation populaire est en phase avec cette démarche. «Plus tard, de 1906 à 1918, Rosier représente le parti au Conseil d'État, organe exécutif du Canton de Genève. Ses grandes victoires sont liées à l'amélioration d'une école qui vise à être la plus égalitaire possible. $»^{8}$

11 Donc, comme le rappelle l'actuelle conservatrice du Département des Cartes et Plans, Marianne Tsioli :

en 1893, Perron dépose les six mille huit cent treize cartes réunies dans quarantetrois portefeuilles à la Bibliothèque où elles constitueront la collection cartographique. En 1902, il y ajoutera deux cent quarante-cinq cartes, quatre-vingt photos et quarante et un reliefs. Perron et Reclus joignent à ce don nombre d'ouvrages de géographie et d'atlas, afin de promouvoir l'étude de la géographie."

\section{La cartographie et les enjeux de la troisième dimension du monde}

12 Mais le projet ne se concrétise pas tout de suite. Charles Perron, en attendant de pouvoir réaliser la mise à disposition publique de ce fonds, expérimente avec Reclus (qui s'est désormais installé en Belgique, à l'Université Nouvelle de Bruxelles), les expressions de qu'on appelle alors la " cartographie nouvelle ».

13 Conscients des enseignements de Carl Ritter sur l'insuffisance de la carte géographique bidimensionnelle comme représentation du monde, et du monopole exercé alors par les armées et par les États sur la cartographie topographique, les géographes anarchistes développent une critique de la carte plane, qui les pousse à expérimenter la construction de représentations tridimensionnelles du monde.

14 L'exemple le plus célèbre de cette démarche est le projet du Grand Globe à l'échelle du cent-millième, présenté par Reclus pour l'exposition universelle de 1900 à Paris. D'après Reclus,

le globe l'emporte sur la carte par le caractère de vérité : il représente la planète dans sa véritable structure, se module exactement sur les vrais contours, tandis que les cartes, d'autant plus fausses qu'elles s'appliquent à une partie plus considérable de la surface planétaire, ne peuvent que tromper le lecteur sur les dimensions 
relatives des régions différentes [tandis que] sur la rondeur d'un globe artificiel aucune méprise n'est possible au sujet de la superficie relative des diverses individualités terrestres. ${ }^{10}$ beaucoup étudiée, ${ }^{11}$ sinon pour souligner que les travaux de Perron en Suisse, dans les années 1890 , s'encadrent dans ce même projet. Perron travaille à un relief au $100.000^{\mathrm{e}}$ de la Suisse, qui n'est autre chose que le premier morceau de l'énorme globe en plâtre, de 127,5 mètres de diamètre. Un travail parallèle est alors en cours en Ecosse, sous la direction de Patrick Geddes, ami de Reclus et soutien enthousiaste du Grand Globe. Reclus écrit à Perron :

Quel beau fragment de notre Globe serait la Suisse [...] Votre Suisse que vous dériverez sans doute par des épreuves de montagne prises sur le modèle primitif, sera dès maintenant le gros morceau d'attaque. Il avait été convenu, je crois, que l'appel serait signé de moi seul. Dès que je serai en mesure de passer à l'exécution, nous paraîtrons dans notre trinité [...] Si cette carte d'Écosse commence, sans doute par le petit fragment Édimbourg-Glasgow, elle portera, si cela ne vous paraît pas prématuré, la mention - Fragment du Globe projeté par E.R., Ch. P. et P.R. ${ }^{12}$

Les reliefs visent à construire une représentation des trois dimensions du monde reproduisant de façon la plus possible correcte la courbure terrestre et surtout présentant les hauteurs à la même échelle que les longueurs, tandis que la plupart des reliefs de l'époque exagéraient les dimensions relatives des montagnes pour pouvoir les rendre percevables à la petite échelle. Cela implique l'utilisation de la grande échelle, en tout cas plus grande du millionième (généralement, de 1/500.000 à 1/5.000).

En 1894, Perron présente son premier projet de relief de la Suisse au bureau de la SGG, qui en accepte le principe tout en précisant que son soutien ne pourra être que « moral». «M. Perron préparera, pour l'exposition de 1896, une carte relief de la Suisse au $500.000^{e}$ d'après sa méthode. Il a demandé à la Société de s'intéresser à la chose : il invite le Bureau à venir visiter le travail. Le Président lui a répondu que la Société lui fera peut-être une allocation, qui ne pourrait d'ailleurs être qu'une bagatelle, et qu'elle s'intéresserait moralement à la chose. $»^{13}$ En tout cas, «M. Perron remercie la Société pour sa souscription éventuelle à la carte projetée, et accepte la condition de donner le quart du revenu une fois les dépenses payées. $»^{14}$

Perron ne participera pas à l'Exposition de Genève de 1896, mais en revanche il commence à travailler à son relief au $100.000^{\mathrm{e}}$ pour l'Exposition de Paris, tout en fixant les règles de sa «nouvelle cartographie »:

1. Les reliefs ont pour objet de montrer la configuration du sol telle qu'elle est. 2. Ils ne doivent admettre aucune des conventions en usage dans les cartes de géographie. 3. Rien ne doit y être représenté qui ne soit à l'échelle. 4. Les reliefs représentant tout ou partie de l'écorce terrestre doivent en avoir la courbure exacte. 5. Les reliefs doivent être construits selon des procédés mécaniques assez précis pour que l'exactitude mathématique en soit la résultante. 6. Les reliefs rentrent dans le domaine des sciences exactes où l'art ne doit intervenir qu'en seconde ligne. ${ }^{15}$

Le principe scientifique de cette proposition est accepté par la SGG, dont le secrétaire, Arthur de Claparède, donne une communication consacrée aux reliefs de Perron au Congrès International de Géographie, tenu à Berlin en 1900. "Le grand avantage du relief est de compléter les cartes en montrant la surface de la terre sous sa forme vraie, ce que ne peuvent faire celles-ci avec les nombreuses conventions qu'il est de leur nature même d'admettre. C'est ainsi que, de l'avis des plus grands géographes, elles 
nous inoculent des idées erronées que les reliefs sont précisément appelés à détruire ou à prévenir. $»^{16}$

Entre-temps, le projet du Grand Globe échoue par manque de financements, tandis que Perron, d'accord avec Reclus, continue le relief de la Suisse pour le présenter à l'Exposition indépendamment du Globe. Nous savons, par une longue lettre qu'il écrit au peintre genevois et sympathisant libertaire Daniel Baud-Bovy, que Perron avait obtenu une allocation de 1.500 francs du Conseil fédéral suisse, ensuite révoquée à cause de l'intervention d'un groupe de cartographes zurichois, jaloux du succès de ce cartographe genevois et en plus anarchiste. Trouver un soutien financier à Genève fut compliqué car, d'après Perron, «ils sont peu prêteurs nos aristocrates genevois. $»^{17}$ Seule, remarquable exception le chocolatier Suchard, qui lui offre une allocation de 5.000 francs à titre de mécénat, après avoir été informé des démarches entamées en Suisse alémanique contre le cartographe. Ensuite, il ne reste que de se mettre à la besogne : comme Perron doit terminer son relief tout seul, son régime de travail dans ces années rappelle le proverbial style de vie de Reclus. « Je devais travailler 15 heures par jour, jours et dimanches, veiller continuellement jusqu'à dix heures. En hiver me levai à 4 heures, allumais ma lampe et mon poêle et me trouvais à le besogne à cinq heures. $»^{18}$ Reclus, de Bruxelles, ne manque pas d'encourager son camarade : «Quoique votre affaire soit retardée, il ne me semble pas qu'elle soit enterrée: tout naturellement, vous avez à la poursuivre avec une persévérance inlassable. $»^{19}$

21 Le relief participe enfin à l'exposition de Paris, en gagnant une médaille d'or. À Genève, cette paroi de plâtre représentant en relief toute la Suisse est exposée avec tous les honneurs dans le bâtiment électoral du 26 décembre 1900 au 13 janvier 1901, pour trouver enfin une collocation permanente dans l'atrium de l'Université de Genève.

Dans la même période Reclus, désormais âgé et malade, travaille à son dernier essai de représentation du monde dans ses trois dimensions: les atlas globulaires, ou cartes sphériques, que nous citons pour leur implication dans le projet du Musée. Il s'agit de feuilles recourbées d'aluminium, dont la surface est lisse mais dont la convexité arrive à reproduire la courbure terrestre à la petite échelle, notamment $1 / 5000000$. Donc, ils représentent les différents pays dans leurs vraies proportions, au contraire des atlas et des planisphères conventionnels. D'après Reclus, si on se passe des océans, à cette échelle 50 feuilles de 46 centimètres de côté suffiraient à représenter toute la planète dans un seul Atlas, dont les dimensions seraient un peu encombrantes, mais toujours gérables pour un emploi didactique, car les feuilles peuvent s'emboiter l'une sur l'autre et être ainsi transportées.

En effet, l'enjeu de ces représentations est encore une fois pédagogique : ces plaques servent pour les salles de cours, à partir du primaire. C'est à l'âge de l'éducation primaire, d'après Reclus, que les cartes bidimensionnelles sont le plus dangereuses, car elles trompent l'intelligence de l'enfant par l'inoculation de modèles erronés: le géographe affirme en 1903, devant l'assemblée de la Royal Geographical Society de Londres, que la carte plane, au niveau du primaire, serait «à interdire complètement. $»^{20}$ Après avoir présenté ce dernier projet à Londres et à Berlin, Reclus meurt en 1905 sans avoir pu achever son Atlas globulaire, mais les premières plaques d'essai, construites par Émile Patesson, sont envoyées en Suisse, où Perron les inclura dans son recueil. Ces expérimentations cartographiques, incluant la production de petits globes de carton pour les salles de cours de l'école primaire, témoignent des relations directes que l'Université Nouvelle de Bruxelles entretient avec les écoles 
libertaires actives à l'époque, notamment la Escuela Moderna de Barcelone, dirigée par Francisco Ferrer y Guardia. ${ }^{21}$

\section{L'exposition : stratégies de communication}

Le nouveau prestige acquis par Perron suite à l'Exposition Universelle de 1900 contribue sans doute au succès de ses efforts volontaristes et opiniâtres pour le musée cartographique : «Perron, nommé officiellement conservateur en 1903, entreprend le classement méthodique et le catalogage de la collection et il obtiendra en outre un crédit spécial [...] En 1905, le Dépôt des cartes de la Ville de Genève s'installe donc dans le bâtiment des Bastions. $»^{22}$

Suite à l'intéressement du Conseil administratif de la Ville de Genève, les institutions rédigent aussi un appel à donations pour compléter et mettre à jour le fonds. D'après Perron, « cet appel eut un plein succès. C'est ainsi qu'en 1902 et 1903, la mappothèque s'augmente de plus de mille cartes, de nombreux atlas, cartes murales etc., provenant en majeure partie de dons. ${ }^{23}$ En 1904, le cartographe présente à la SGG l'état de cette « mappothèque » :

On y trouve pour chaque pays, en outre des cartes d'État-major ou autres cartes générales, de nombreuses cartes régionales, plans de ville, etc., ainsi que des cartes se rapportant à l'archéologie, à l'ethnographie, à la politique, à la guerre. Puis encore d'autres relatives à la géologie, à la production naturelle du sol, à l'agriculture, à l'industrie, au commerce, aux voies de communication, à la statistique, etc. Un assez grand nombre de cartes portent des annotations de la main de M. Reclus, ce qui n'est pas pour en diminuer la valeur [...] Notre mappothèque contient encore d'autres documents, parmi lesquels se trouvent, en assez grand nombre, des cartes spéciales avec dédicace de leurs auteurs, des cartes manuscrites d'explorateurs; d'autres, également manuscrites, de cartographes connus comme Vuillemin, de graveurs célèbres comme Collin; enfin, quelques pièces rares..$^{24}$

Le 14 novembre 1907, le Musée Cartographique de la Ville de Genève ouvre ses portes, à la présence de Perron, Rosier, De Claparède et du conseiller de la Ville de Genève Piguet-Fages, qui prononce un discours d'inauguration. ${ }^{25}$ Comme le remarque Marianne Tsioli, cette « initiative privée a provoqué un véritable bouleversement, transformant une honnête collection de bibliothèque encyclopédique en ensemble unique et exceptionnel, aux racines de la géographie moderne. ${ }^{26}$ Le même auteur envisage très clairement la continuité pédagogique entre la NGU, avec laquelle « Reclus et Perron visaient à mettre un savoir géographique à la disposition de tous $»,{ }^{27}$ et le Musée Cartographique, conçu pour "intéresser le grand public [...] tout en facilitant les travaux des hommes d'étude. $»^{28}$

27 L'exposition, n'intégrant qu'une partie de l'énorme fonds cartographique, est organisée en cinq séries principales: les mappemondes (176 pièces); l'histoire du dessin cartographique ( 55 pièces); les cartes marines ( 30 pièces); les cartes de la Suisse (50 pièces); les cartes du canton et de la ville de Genève ( 40 pièces), plus une section de "cartes diverses" (10 pièces). Comme exemple de la "nouvelle cartographie », un disque globulaire de Reclus représentant la Méditerranée occidentale (figure 1) est placé à la fin de la section d'histoire du dessin cartographique pour rappeler, comme Reclus l'écrivait à Perron, «que la terre est ronde et que les cartes logiques doivent l'être aussi. ${ }^{29}$ 
Figure 1: Élisée Reclus et Émile Patesson, Carte Globulaire de la Méditerranée Occidentale (Bruxelles, 1903)

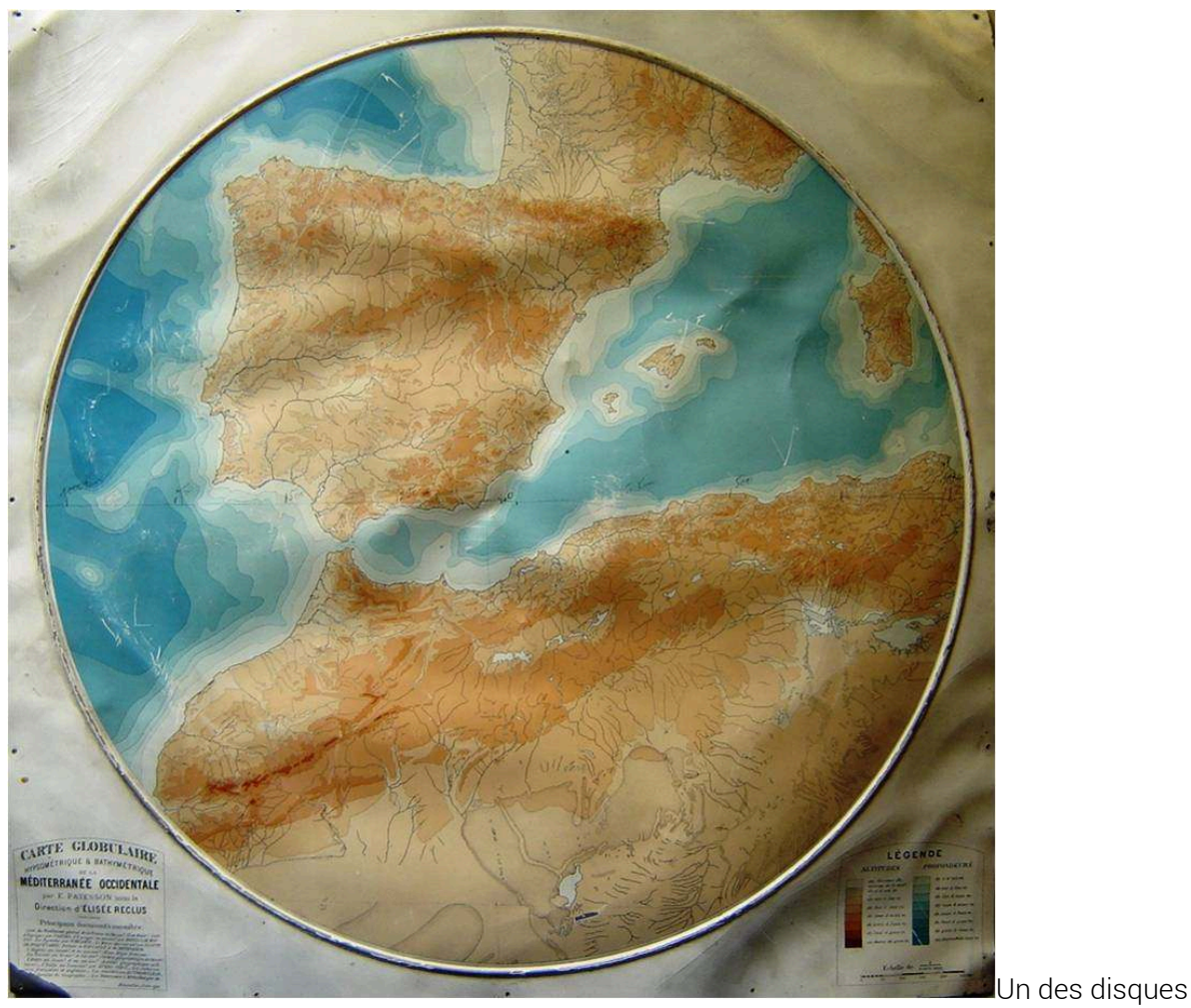

globulaires déposés à la BGE (Bibliothèque de Genève, Département de Cartes et Plans), fait en aluminium, échelle de 1:5000 000.

Photographie de l'auteur.

Deux publications accompagnent l'ouverture du Musée Cartographique. La première est son catalogue (figure 2), comprenant une fiche descriptive synthétique de chaque carte exposée, et une courte introduction pour chaque section de l'exposition, qui ne doit pas rentrer dans les détails techniques. « Le catalogue ne traitera pas des méthodes successivement employées dans la construction des cartes, non plus que des recherches relatives aux distinctions épineuses des sources, des écoles, etc., ces questions analytiques étant trop spéciales et ardues pour trouver place dans un simple exposé mis, autant que possible, à la portée de tous. ${ }^{30}$ 
Figure 2: Frontispice du Catalogue Descriptif du Musée Cartographique (1907)

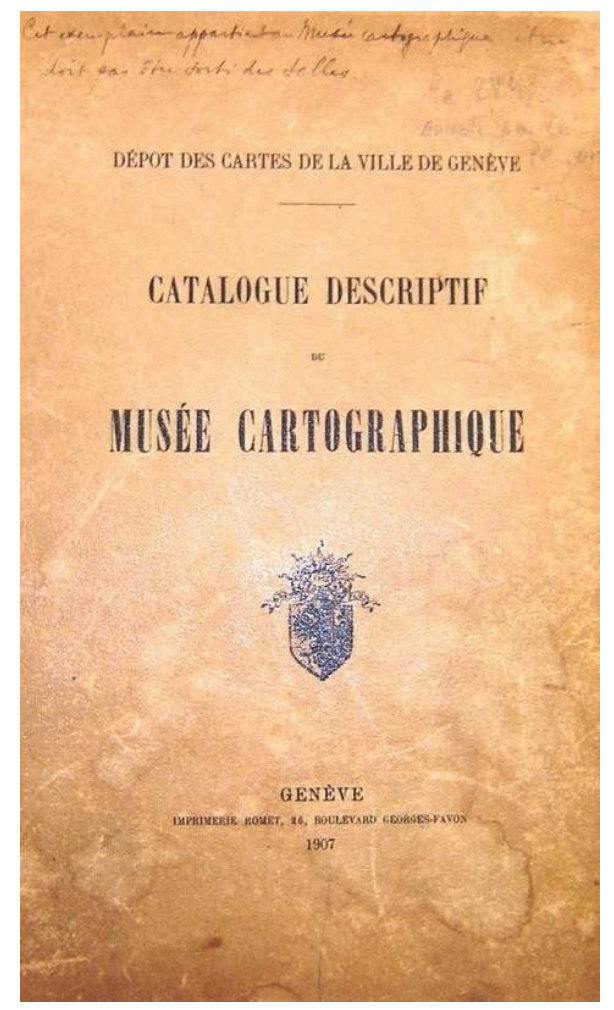

Bibliothèque de Genève (BGE).

Photographie de l'auteur

La deuxième est un ouvrage de Perron, publié à Paris par la Revue des Idées, sur l'histoire des mappemondes de l'antiquité jusqu'au $19^{\mathrm{e}}$ siècle, où le souci pédagogique prime encore :

Je voudrais être réussi à faire comprendre, au moins en partie, l'importance que les musées cartographiques pourraient avoir pour les études scientifiques comme pour l'instruction publique. Ne suffit pas, en effet, de connaître l'existence des vieux documents de l'histoire de la cartographie, il faudrait que, comme les tableaux dans les galléries d'art, ils fussent accessibles à tous. Cachés dans des cartons, leur utilité est des plus restreintes, puisqu'on ne les en retire, à un à un, que lorsque d'aventure quelque érudit en fait la demande. Cela n'est pas suffisant. Quels travaux, outre que ceux d'analyse, peut produire la consultation de documents isolés? Les études comparées que permettrait leur vue simultanée auraient aussi leur valeur, sans doute. Et puis, dans notre siècle d'instruction démocratique, ne devrait-on pas mettre en lumière l'œuvre humaine peut-être la plus grande et la plus importante de toutes, celle qui, commencée dans le profond éloignement des siècles, se poursuit encore de nos jours avec passion : la découverte de la Terre ? $^{31}$

Perron s'engage aussi, au sein de la SGG, pour que la proposition d'ouvrir des nouveaux musées cartographiques soit portée au Congrès International de Géographie prévu en 1908 à Genève. Comme le témoignent les procès-verbaux manuscrits de son Bureau, récemment ouverts à la consultation à la BGE (tandis que les séances plénières sont publiées dans la revue Le Globe), la Société retient cette proposition.

Dans un entretien qu'il a eu avec le Président, comme suite à la décision du Bureau de 7 juin, M. Perron a expliqué qu'il vaudrait: 1) que le congrès décidât de faire faire des facsimilés des cartes rares anciennes pour les conserver et permettre la formation de musées ; 2) qu'un organisme fut constitué pour l'étude des voies et 
moyens et que, dans ce but, la rédaction d'un projet à présenter au Congrès soit préparée par la Société de Géographie. Décidé d'examiner les propositions à faire au Congrès dans le sens qui précède..$^{32}$

Perron participe à ce Congrès, où il est nommé membre d'une Commission chargée d'examiner les conditions pour la reproduction et la mise à disposition publique des «monuments cartographiques de l'humanité ».

Le Neuvième Congrès International de Géographie émet le vœu que les sociétés de géographie veuillent bien chercher d'intéresser les gouvernements de leurs pays respectifs à la réfection des monuments cartographiques de l'Antiquité, du Moyen Age et de la Renaissance, documents d'une grande valeur scientifique, et que le temps menace de détruire. Le Congrès nomme MM. Nordenskjöld, K. Miller, G. Marcel, E. Oberhummer et C. Perron membres d'une Commission ayant pour mandat de centraliser les résultats obtenus dans cet ordre d'idées, de présenter au prochain congrès un catalogue donnant l'état général de la réfection des cartes anciennes en fac-similé, et de déterminer par ordre d'importance les documents cartographiques anciens dont la restitution serait particulièrement désirable. La Commission pourra s'adjoindre, par cooptation, des membres appartenant aux divers pays possédant des documents cartographiques. ${ }^{33}$

Parmi les membres cooptés nous trouvons un cartographe français et auteur de manuels de géographie scolaire, Franz Schrader, qui est aussi le cousin de Reclus et l'un des dernières survivants, en ce moment, de l'ancien réseau des collaborateurs de la NGU. ${ }^{34}$

Perron meurt en 1909, mais le Musée reste ouvert sous la direction de Charles Schöndelmayer : «à cette époque, plus de six cents personnes visitaient chaque année le Musée Cartographique ${ }^{35}$. Cependant, une fois décédé son animateur infatigable, le Musée décline graduellement : le nombre de visiteurs baisse, les heures d'ouverture se réduisent, et cette institution est fermée en 1922 " par mesure d'économie. »"

\section{Conclusion}

Si l'histoire du Musée Cartographique relève clairement de l'effort volontariste d'un géographe hétérodoxe comme Perron, militant anarchiste et survivant du réseau de militants et exilés qui avaient animé la Fédération Jurassienne tout en travaillant à la NGU, elle s'insère néanmoins, malgré son exceptionnalité, dans des conditions historiques très précises.

D'abord, on est dans la période où l'on construit des institutions éducatives nationales destinées à servir toute la population: comme nous l'avons dit, les anarchistes collaborent avec les avant-gardes du mouvement de l'éducation populaire et laïque, comme William Rosier dans le cas de Genève. Le mouvement des musées et des expositions s'insère dans ce contexte culturel; ce n'est pas un hasard si la Ville de Genève a déjà une Délégation aux expositions et musées (dont le responsable est Piguet-Fages) avec laquelle Perron peut dialoguer.

La spécificité suisse et genevoise joue aussi son rôle. Genève a été pendant plusieurs siècles le lieu de refuge d'exilés politiques de toute l'Europe : c'est dans les années 1870 que la rencontre en terre helvétique entre les exilés russes et slaves persécutés par le régime tzariste et les Français réfugiés après la Commune de 1871 a rendu possible à la fois la constitution du mouvement anarchiste organisée ${ }^{37}$ et l'établissement du réseau qui a travaillé à la NGU. 

déposé au Département des Cartes et Plans de la Bibliothèque de Genève, où il n' accessible que sur rendez-vous. Cependant, dans les dernières années l'attention des chercheurs et des institutions s'est accrue, et le projet Écrire le Monde Autrement, débutant en septembre 2012 au Département de Géographie de l'Université de Genève, [http://www.unige.ch/ses/geo/index.html], se propose de commencer l'exploitation de ce recueil de sources dont l'analyse n'est qu'à ses débuts.

\section{BIBLIOGRAPHIE}

ALAVOINE-MULLER S. Un globe terrestre pour l'Exposition universelle de 1900. L'utopie géographique d'Élisée Reclus. L'Espace géographique. v. 31, p. 156-170, 2003.

CODELLO Francesco. La buona educazione: esperienze libertarie e teorie anarchiche in Europa da Godwin a Neill. Milano: Angeli, 2005.

DUBOIS Patrick. Le Dictionnaire de pédagogie et d'instruction primaire de Ferdinand Buisson : répertoire biographique des auteurs. Paris-Lyon-Rouen : Institut National de recherche pédagogique, 2002.

DUNBAR G. Élisée Reclus and the Great Globe. In DUNBAR Gary (ed.). The History of Geography. New York: Dodge-Graphic Press Inc., 1996, p. 12-22. 
ENCKELL M. Élisée Reclus inventeur de l'anarchisme. In CREAGH Ronald et alii (ed.). Élisée Reclus - Paul Vidal de la Blache. Le géographe, la cité et le monde, hier et aujourd'hui. Autour de 1905. Paris: L'Harmattan, 2009, p. 39-44.

FERRETTI Federico. Il mondo senza la mappa, Élisée Reclus e i geografi anarchici. Milano: Zero in Condotta, 2007.

FERRETTI F. Charles Perron, le cartographe de la "juste représentation du monde". Visions Cartographiques/Le Monde Diplomatique, 2010, [http://blog.mondediplo.net/2010-02-05-CharlesPerron-cartographe-de-la-juste].

FERRETTI Federico. Anarchici ed editori, reti militanti, editoria e lotte culturali attorno alla Nuova Geografia Universale di Elisée Reclus. Milano : Zero in Condotta, 2011.

FERRETTI F. L'Occident d'Élisée Reclus, l'invention de l'Europe dans la Nouvelle Géographie Universelle. Paris : 2011. Thèse soutenue sous la direction de Marie-Claire Robic.

FISCHER C., MERCIER C., RAFFESTIN C. Entre la politique et la science, un géographe genevois : William Rosier. Le Globe, v. 143, p. 13-25, 2003.

JANKOVIC N. Introduction. In RECLUS Élisée. Projet de globe au 100.000. Paris : Éditions B2, 2011, p. 7-41.

JUD Peter. Élisée Reclus und Charles Perron, Schöpfer der "Nouvelle Géographie Universelle" : ein Beitrag zur geographischen Wissenschaftshistoire des 19. Jahrhunderts. Zürich, 1987.

PELLETIER Philippe. Élisée Reclus, géographie et anarchie. Paris : Éditions du Monde Libertaire, 2009.

REUBI Serge. Gentlemen, prolétaires et primitifs : institutionnalisation, pratiques de collection et choix muséographiques dans l'ethnographie suisse, 1880-1950. Bern, Lang, 2011.

TSIOLI BONDENMANN M. Cartes et Plans. In Patrimoines de la Bibliothèque de Genève. Genève, Slatkine, 2006, p. 189-197.

WALTER François. Les figures paysagères de la nation, territoire et paysage en Europe $\left(16^{\mathrm{e}}\right.$ $\mathbf{2 0}^{\mathrm{e}}$ siècle). Paris : Éditions de l'EHESS, 2004.

\section{ANNEXES}

\section{Arquivos}

Bibliothèque de Genève (BGE) - Département des Manuscrits ; Département des Cartes et Plans.

Paris - Institut Français d'Histoire Sociale (IFHS), 14 AS 232, Correspondance d'Élisée Reclus.

\section{Fontes impressas}

DE CLAPARÈDE A. Un Nouveau Procédé de construction des reliefs employé par M. C. Perron, Cartographe à Genève. In Sonderabdruck aus den Verhandlungen des VII Internationalen Geographen-Kongresses in Berlin, 1899. Berlin: 1900, p. 941-945.

GUILLAUME James. L'Internationale, documents et souvenirs. Paris : Lebovici, 1985. 
Neuvième Congrès International de Géographie. Genève, 27 juillet - 6 août 1908.

Résolutions et vœux votés par l'assemblée des délégués le lundi 3 août et le jeudi 6 août 1908. Genève : Société Générale d'imprimerie, 1908 [Annexe au tome XLVIII du Globe].

PERRON Charles. De l'obligation en matière d'instruction. Genève : Imprimerie Vaney, 1868.

PERRON Ch. La bibliothèque cartographique de M. Élisée Reclus. Le Globe. Organe de la Société de Géographie de Genève. v. 30, p. 162-163, 1891.

PERRON Charles. Des reliefs en général et du relief au $100.000^{\mathrm{e}}$ de la Suisse en particulier, mémoire adressé au Jury de la Cartographie à l'Exposition universelle de 1900 à Paris. Genève : Stampelhor, 1900.

PERRON Ch. Collection Cartographique de la Bibliothèque Publique. Le Globe. Organe de la Société de Géographie de Genève. v. 43, p. 38-45, 1904.

PERRON Charles. Catalogue descriptif du Musée cartographique / Dépôt des cartes de la Ville de Genève. Genève : Imprimerie Romet, 1907.

PERRON Charles. Une étude cartographique. Les Mappemondes. Paris : Éd. de la Revue des Idées, 1907.

RECLUS Élisée. Projet de construction d'un globe terrestre à l'échelle du cent-millième. Paris : Edition de la Société Nouvelle, 1895.

RECLUS É. On spherical maps and reliefs. The Geographical Journal. n. 3, p. 290-293, 1903.

\section{NOTES}

1. FERRETTI F. Charles Perron, le cartographe de la "juste représentation du monde". Visions Cartographiques/Le Monde Diplomatique, 2010, [http://blog.mondediplo.net/2010-02-05-CharlesPerron-cartographe-de-la-juste].

2. FERRETTI Federico. Anarchici ed editori, reti militanti, editoria e lotte culturali attorno alla Nuova Geografia Universale di Elisée Reclus. Milano : Zero in Condotta, 2011; JUD Peter. Élisée Reclus und Charles Perron, Schöpfer der "Nouvelle Géographie Universelle" : ein Beitrag zur geographischen Wissenschaftshistoire des 19. Jahrhunderts. Zürich, 1987; PELLETIER Philippe. Élisée Reclus, géographie et anarchie. Paris: Éditions du Monde Libertaire, 2009.

3. PERRON Ch. La bibliothèque cartographique de M. Élisée Reclus. Le Globe. Organe de la Société de Géographie de Genève. v. 30, p. 162, 1891.

4. CODELLO Francesco. La buona educazione: esperienze libertarie e teorie anarchiche in Europa da Godwin a Neill. Milano: Angeli, 2005.

5. PERRON Charles. De l'obligation en matière d'instruction. Genève : Imprimerie Vaney, 1868.

6. GUILLAUME James. L'Internationale, documents et souvenirs. Paris : Lebovici, 1985, vol. IV, p. 147.

7. DUBOIS Patrick. Le Dictionnaire de pédagogie et d'instruction primaire de Ferdinand Buisson: répertoire biographique des auteurs. Paris-Lyon-Rouen: Institut National de recherche pédagogique, 2002.

8. FISCHER C., MERCIER C., RAFFESTIN C. Entre la politique et la science, un géographe genevois : William Rosier. Le Globe, v. 143, p. 17, 2003. 
9. TSIOLI BONDENMANN M. Cartes et Plans. In Patrimoines de la Bibliothèque de Genève. Genève : Slatkine, 2006, p. 191.

10. É RECLUS Élisée. Projet de construction d'un globe terrestre à l'échelle du cent-millième. Paris : Edition de la Société Nouvelle, 1895, pp. 3-4.

11. ALAVOINE-MULLER S. Un globe terrestre pour l'Exposition universelle de 1900. L'utopie géographique d'Élisée Reclus. L’Espace géographique. v. 31, p. 156-170, 2003; DUNBAR G. Élisée Reclus and the Great Globe. In DUNBAR Gary (ed.). The History of Geography. New York: DodgeGraphic Press Inc., 1996, p. 12-22; FERRETTI Federico. Il mondo senza la mappa, Élisée Reclus e i geografi anarchici. Milano: Zero in Condotta, 2007; JANKOVIC N. Introduction. In RECLUS Élisée. Projet de globe au 100.000. Paris : Éditions B2, 2011, p. 7-41.

12. Bibliothèque de Genève (dorénavant BGE), Dép. des Manuscrits, Ms. Suppl. 119, lettre d'E. Reclus à Ch. Perron, 1 déc. 1895 [P.R. sont les initiales de Paul Reclus, ingénieur, neveu d'élisée et chargé de la partie technique du projet].

13. BGE, Dép. des Manuscrits, Ms. Fr. 7996/2, Société de Géographie, Procès-verbaux des séances du Bureau, 1890-97, séance du 4 avril 1894.

14. Ibid., séance du 15 mai 1894.

15. PERRON Charles. Des reliefs en général et du relief au $\mathbf{1 0 0 . 0 0 0}^{\mathrm{e}}$ de la Suisse en particulier, mémoire adressé au Jury de la Cartographie à l'Exposition universelle de 1900 à Paris. Genève : Stampelhor, 1900, pp. 7-8.

16. DE CLAPARÈDE A. Un Nouveau Procédé de construction des reliefs employé par M. C. Perron, Cartographe à Genève. In Sonderabdruck aus den Verhandlungen des VII Internationalen Geographen-Kongresses in Berlin, 1899. Berlin: 1900, p. 941.

17. BGE, Dép. des Manuscrits, Archives Baud-Bovy 270/4, ff. 146-148, lettre de Ch. Perron à D. Baud-Bovy, 5 fév. 1901.

18. Ibid.

19. Institut Français d'Histoire Sociale (IFHS), 14 AS 232, Correspondance d'Élisée Reclus, lettre d'É. Reclus à Ch. Perron, 17 juil. 1897.

20. RECLUS É. On spherical maps and reliefs. The Geographical Journal. n. 3, p. 290, 1903.

21. FERRETTI Federico. Il mondo senza la mappa, cit.

22. TSIOLI BONDENMANN M. Cartes et Plans, cit. p. 191.

23. PERRON Ch. Collection Cartographique de la Bibliothèque Publique. Le Globe. Organe de la Société de Géographie de Genève. v. 43, p. 41, 1904.

24. Ibid., p. 39.

25. BGE, Dép. des Manuscrits, Biographies Genevoises, 1909, Charles Perron.

26. TSIOLI BONDENMANN M. Cartes et Plans, cit. p. 189.

27. Ibid., p. 190.

28. PERRON Charles. Catalogue descriptif du Musée cartographique / Dépôt des cartes de la Ville de Genève. Genève : Imprimerie Romet, 1907, p. 3.

29. BGE, Dép. des Manuscrits, Ms. Suppl. 119, lettre d'E. Reclus à Ch. Perron, 9 nov. 1902.

30. PERRON Charles. Catalogue descriptif du Musée cartographique, cit., p. 5.

31. PERRON Charles. Une étude cartographique. Les Mappemondes. Paris : Éd. de la Revue des Idées, 1907, p. 44.

32. BGE, Dép. des Manuscrits, Ms. Fr. 7996/3 Société de Géographie, Procès-verbaux des séances du Bureau 1907-1916, Séance du 5 juillet 1907.

33. Neuvième Congrès International de Géographie. Genève, 27 juillet - 6 août 1908. Résolutions et vœux votés par l'assemblée des délégués le lundi 3 août et le jeudi 6 août 1908. Genève : Société Générale d'imprimerie, 1908, p. 22-23.

34. FERRETTI F. L'Occident d'Élisée Reclus, l'invention de l'Europe dans la Nouvelle Géographie Universelle. Paris : 2011. Thèse soutenue sous la direction de Marie-Claire Robic.

35. TSIOLI BONDENMANN M. Cartes et Plans, cit., p. 191. 
36. Ibid., 192.

37. ENCKELL M. Élisée Reclus inventeur de l'anarchisme. In CREAGH Ronald et alii (ed.). Élisée Reclus - Paul Vidal de la Blache. Le géographe, la cité et le monde, hier et aujourd'hui. Autour de 1905. Paris: L'Harmattan, 2009, p. 39-44.

38. WALTER François. Les figures paysagères de la nation, territoire et paysage en Europe $\left(\mathbf{1 6}^{\mathrm{e}}-\mathbf{2 0}^{\mathrm{e}}\right.$ siècle). Paris : Éditions de l'EHESS, 2004.

39. REUBI Serge. Gentlemen, prolétaires et primitifs : institutionnalisation, pratiques de collection et choix muséographiques dans l'ethnographie suisse, 1880-1950. Bern, Lang, 2011.

\section{RÉSUMÉS}

Cet article aborde la constitution du Musée Cartographique de la Ville de Genève, ouvert entre 1907 et 1922 à partir du fonds cartographique de Charles Perron et d'Élisée Reclus. À travers l'analyse de sources édites et inédites, nous reconstruisons cette expérience en nous interrogeant sur le rapport entre géographie et éducation populaire, et sur le rôle de la cartographie dans la démarche de ces géographes.

Reclus, histoire de la cartographie, histoire de l'éducation géographique

Este artigo trata da constituição do Museu Cartográfico da Cidade de Genebra, aberto de 1907 a 1922 a partir do fundo cartográfico de Charles Perron e Élisée Reclus. Nos reconstruímos esta experiência pela análise de fontes publicadas e inéditas, problematizando a relação entre geografia e educação popular, e o papel da cartografia nas concepções científicas destes geógrafos.

Este artículo trata de la constitución del Museo Cartográfico de la Ciudad de Ginebra, abierto entre 1907 y 1922 con base en el fondo cartográfico de Charles Perron y Élisée Reclus. Reconstruimos esta experiencia a través del análisis de fuentes publicadas e inéditas, problematizando la relación entre geografía y educación popular, y el papel de la cartografía en las concepciones científicas de estos geógrafos.

This paper deals with the foundation of the Cartographic Museum of Geneva, open from 1907 to 1922 and based on the cartographic collection of Charles Perron and Élisée Reclus. We reconstruct this experience by the analysis of edited and unedited sources, questioning the relationship between geography and popular education, and the role of cartography in the scientific strategy of these geographers.

\section{INDEX}

Keywords : Reclus, history of cartography, history of geographic education

Index géographique : Genève

Index chronologique : 1907,1922

Palabras claves : Reclus, historia de la cartografía, historia de la educación geográfica

Palavras-chave : Reclus, história da cartografia, história da educação geográfica 
AUTEUR

FEDERICO FERRETTI

Docteur en Géographie, chercheur à l'Université de Genève, Département de Géographie et Environnement, membre de l'UMR 8504 Géographie-cités, équipe EHGO.

federico.ferretti@unige.ch 